\section{AN UNUSUAL CASE OF ADRENAL CARCINOMA}

\section{WITH A NOTE ON THE APPLICATION OF A NEW} COLOUR TEST

BY

\author{
L. R. BROSTER, D.M., M.Ch., F.R.C.S. \\ AND
}

JOCELYN PATTERSON, M.Sc., Ph.D., F.R.S.Ed. (From Charing Cross Hospital)

This case is recorded not only because it was unique in our series in having general clinical appearances of a typical Group I virilism associated with an adreno-cortical tumour instead of marked bilateral hyperplasia, but also because the later stages were accompanied by manifestations of disturbances in both carbohydrate and steroid metabolism of a particularly striking order. Moreover, the case afforded an opportunity of applying the urinary colour test recently devised by Patterson (1947) for distinguishing between adreno-cortical tumour and marked adrenal hyperplasia.

\section{Case Report}

The patient, a girl aged $14 \frac{1}{2}$ years, was kindly referred by Dr. H. B. Hayman, of Gloucester. He wrote : "She had been admitted to the City General Hospital, Gloucester, on April 20,1947, with a four-months history of fits followed by coma. During the past 18 months she had become very hairy, the voice had deepened, her secondary sex characters had not appeared, and she had never menstruated. On admission she was semicomatose, with a marked right-sided hemiplegia and all the signs of an upper-motor-neurone lesion. The latter completely disappeared within the next three days. She then developed epileptiform fits accompanied by incontinence of urine. Each fit lasted 30 minutes, with a return to normal. The fits then began to increase in frequency, with lengthening periods of unconsciousness.

"On examination her complexion was dark and the nipples pigmented. She was drowsy and her voice was deep. There was marked hirsuties, also a male pubic triangle. The clitoris was enlarged to the size of the tip of the little finger. The blood pressure was $120 / 85$ and the cardiovascular system was normal. The central nervous system showed on the right side a seventh-nerve palsy, a dilated pupil, and an extensor plantar reflex. The eyes deviated to the left, with papilloedema more pronounced on the right side than on the left. A large tumour was present in the left hypochondrium. The cerebrospinal fluid and urine were normal, the blood urea was $25 \mathrm{mg}$. per $100 \mathrm{ml}$., and the sugar-tolerance test revealed marked hypoglycaemia, with blood-sugar figures following $50 \mathrm{~g}$. of glucose ranging from $55 \mathrm{mg}$. per $100 \mathrm{ml}$. at 15 minutes to $70 \mathrm{mg}$. at 120 minutes, and the urine showing a trace of sugar."

On July 7, 1947, the patient was admitted into Charing Cross Hospital. She had that gnomish appearance typical of marked Group I virilism, with the figure of a boy rather than a girl. The chest was flat, the musculature well developed, with limbs short in proportion to the torso. During lucid periods the mentality appeared dull but psychologically feminine. The blood urea was $19 \mathrm{mg}$. and fasting blood sugar $35 \mathrm{mg}$. per $100 \mathrm{ml}$., haemoglobin $104 \%$, and the 17 -ketosteroid excretion $1,980 \mathrm{mg}$. a day-about $2 \frac{1}{2}$ times the highest level we have seen reported. The dehydroisoandrosterone test (Patterson: see Addendum) was strongly positive. $X$-ray examination showed calcified streaks in a large adrenal shadow, a normal pituitary fossa, and premature epiphysial fusion corresponding to the age of 20 or over. From the day of admission till July 20 , when she died, there was a succession of fits, with stupor and recovery. The stupor would last as long as a whole day. At times she was irritable and incontinent, and on waking would eat ravenously. On July 13 she was roused temporarily by 2 minims $(0.12 \mathrm{ml}$.) of adrenaline intravenously, and on the 14th from a deep stupor by $5 \mathrm{ml}$. of $50 \%$ glucose intravenously. On July 16 she was seen by Dr. Gardiner-Hill, who suggested that the hypoglycaemia was due to the unopposed action of insulin - the adrenal medulla being destroyed-and that she be put on a high-protein lowcarbohydrate diet to avoid the further stimulation of insulin production. During the attack in which the blood sugar was estimated the value fell to $15 \mathrm{mg}$. per $100 \mathrm{ml}$. (Hagedorn and Jensen method). There was no return of any central nervous symptoms, except that during July 19 and 20 the pupils began to dilate with the head turned to the right, and the temperature began to rise.

At necropsy the left adrenal tumour together with attached kidney weighed $6 \mathrm{lb}$. $8 \mathrm{oz}$. $(2.98 \mathrm{~kg}$.). The thyroid gland was enlarged, the pituitary was normal, and there was no evidence of any secondary deposits.

\section{Report on the Tumour}

The following are the findings of Prof. Willis, of the Royal College of Surgeons, with regard to the adrenal cortical tumour.

" Gross Description.-The left adrenal tumour is rounded, well circumscribed, measures $22 \mathrm{~cm}$. in main diameter, and weighs nearly $6 \mathrm{lb}$. $(2.72 \mathrm{~kg}$.) ; it consists of almost fluid degenerated and haemorrhagic material, with only a thin rim of living tumour tissue in places. The kidney and also the other pieces of organs submitted (liver, spleen, thyroid, right adrenal, uterus and ovaries, and pancreas) show no obvious abnormalities, except for marked post-mortem changes.

“Microscopical Description.-The adrenal tumour consists of large, rounded, and irregular cells of variable size, irregularly arranged in masses and trabeculae and showing extensive areas of degeneration. The structure is that of a malignant adrenal cortical tumour, although mitotic figures are not numerous. Spleen, adrenal, kidney, pancreas, liver, ovary, and thyroid show pronounced congestion and post-mortem changes (especially in the pancreas and kidney) and scattered large bacilli which are probably post-mortem invaders. Other noteworthy points are : kidney shows patchy areas of calcification in both the cortex and medulla; post-mortem changes in pancreas are so advanced that islet tissue cannot be distinguished; ovary contained abundance of healthy primary and vesiculat follicles ; and thyroid shows a mild degree of colloid retention."

\section{Discussion}

Clinically the patient did not show such an advanced degree of virilism as other cases of similar age group in our series where the underlying pathology was due to pronounced adrenal hyperplasia. Nevertheless, the 17ketosteroid excretion was 20 to 50 times that commonly found in the latter group. This finding makes the case a notable example of gross discrepancy between clinical manifestations of virilism and the 17-ketosteroid excretion.

It is also a matter of interest that the opposite adrenal was present. This seems to conform to the rule that in heterosexual virilism both adrenals are invariably present, whereas in isosexual precocity the carcinoma may arise in a solitary adrenal. We have experienced the latter combination on three occasions: two boys, one with a tumour imbedded under the right lobe of the liver and one with a huge tumour of the left adrenal; and a girl, also with a huge tumour on the left side, with an androgen excretion of $260 \mathrm{mg}$. a day, who was being treated concurrently with the present case. These patients are of the fat, ruddycomplexioned type, more akin to Cushing's syndrome.

Some of the difficulty of correlating clinical manifestations with 17-ketosteroid output undoubtedly arises from differences in the type of androgen secreted. Crooke (1946) puts forward the view that the association of adreno-genital tumours with virilism in some instances and with basophilism in others might be accounted for on the theory that the androgenic steroids of the first type are derived from incompletely elaborated hormones, whereas in the second type they derive from vital hormones, and that it 
is the excess of these vital hormones which produces atrophy of the opposite adrenal. As Crooke points out, this likewise would help to explain the occasional incidence of Addison's disease in some children of Group I virilism due to bilateral hyperplasia if it be assumed that large amounts of abnormal androgens are being produced at the expense of vital hormones.

The present case, so far as carbohydrate metabolism is concerned, comes more into line with certain cases of Addison's disease than with cases of 'virilism, in which the tendency is in the direction of lowered sugar tolerance. With one adrenal apparently normal, the destruction of the adrenal medulla by the tumour would hardly seem sufficient cause in itself for the marked hypoglycaemia.

In view of the exceptional rate of secretion of androgenic steroids it cannot be excluded that these may have suppressed the anti-insulin action of the right adrenal without causing an atrophy of the gland such as would be expected if the tumour was of the type producing vital hormones.

Some of the present perplexities in this field will most likely be resolved when more clues to the differences in the androgens in clinically distinctive groups are available. The introduction of the relatively simple qualitative dehydroisoandrosterone test is an important step in this direction. As a test of malignancy the potentialities would seem to be not less than those of such acknowledged diagnostic aids as the gonadotrophin assays for chorion epithelioma and the serum acid-phosphatase estimation applied to prostatic carcinoma.

Addendum. - The dehydroisoandrosterone test referred to above is applied to the type of urinary extract used for 17-ketosteroids. The dry extract is first treated with concentrated sulphuric acid at $25^{\circ}$ C. for 20 minutes, the solution diluted with an equal volume of water while being kept cold, and then heated for one minute in a boiling-water bath. A blue or blue-violet colour developing in the final stage indicates the presence of excess dehydroisoandrosterone and its closely related steroids in the extract - a feature of tumour urine extracts but not of the corresponding urinary extracts for cases of adrenal hypertrophy.

In the case described the distinct reaction was readily obtained even when the amount of extract used was reduced to the equivalent of no more than $0.1 \mathrm{ml}$. of original urine.

\section{Summary}

A case is described in which a new test for carcinoma of the adrenal gland has been established.

This test differentiates carcinoma from hyperplasia.

Gross abnormalities of metabolism were present in the terminal stages of the condition.

\section{REFERENCES}

Crooke, A. C. (1946). Proc. R. Soc. Med., 39, 516. Patterson, J. (1947). Lancet, 2, 580.

Unlike the doctors and dentists, pharmacists welcomed the new National Health Service Act, said Mrs. J. K. Irvine, president of the Pharmaceutical Society of Great Britain, speaking in Edinburgh on April 2. Subject to satisfactory terms of service and remuneration they were prepared to take their part in the new service. Under the Act pharmacy became a complete public service applying to the whole population. The new service was going to change pharmacy just as much as the medical benefit part of the Act of 1911 had revolutionized the pharmacy of that day. Pharmacy welcomed the Act because it put an end to the dispensing doctor. Practically all the dispensing would be done by pharmacists. It meant the complete separation of prescribing and dispensing. Pharmacy, so far, was taking the Act on trust, because as yet they had little idea of the terms of service or the remuneration. They hoped that trust would be justified, for the time was now short for any extended or controversial negotiations with the Minister of Health.

\section{ADRENAL FEMINISM DUE TO CARCINOMA OF THE ADRENAL CORTEX}

\section{A CASE REPORT AND REVIEW OF THE LITERATURE}

BY

\section{N. ARMSTRONG, M.D., F.R.C.P., D.P.H.}

AND

JOHN SIMPSON, M.D., M.R.C.P.

(From the Royal Victoria Infirmary, Newcastle-upon-Tyne)

Feminism can be defined as the development of female characteristics in a male. This is usually manifested as a feminine distribution of subcutaneous fat and gynecomastia, often with testicular atrophy and a feminine type of hair distribution. We do not consider that syndromes associated with the failure of development or regression of primary or secondary male characteristics alone as feminism.

Parkes Weber (1926) describes gynecomastia as a rare occurrence in hypogonad states, whether this hypogonadism be primary and possibly due to pituitary insufficiency, or secondary following disease or trauma of the testes. He quotes some remarkable cases, including unilateral gynecomastia following injury or disease of the testes which has itself sometimes been unilateral. Cases of gynecomastia after castration and prostatectomy have been described in the literature. Very rarely, gynecomastia occurs in chorion epithelioma of the testes. Gynecomastia has been reported amongst men engaged in the handling and packing of oestrogen preparations, and has been seen in cases of advanced liver disease, apparently due to the failure of the liver to carry out its role of detoxicating the normally occurring oestrogens.

The most striking group, however, comprises those cases with an adrenal origin. Seven cases of feminism due to carcinoma of the adrenal cortex have been reported-the one described below is the eighth. Broster also describes feminism in cases of hyperplasia of the adrenal cortex, and has performed unilateral adrenalectomy, with improvement. Roholm and Teilum (1942) also discuss the possibility of such a hyperplasia causing feminism.

\section{Review of Recorded Cases}

It is with the clinical picture of feminism due to adrenal neoplasm that this paper is concerned. The first case was described by Bittorf in 1919, and since then Parkes Weber (1926) and zum Busch, Holl (1930 ; 2 cases), Lisser (1936), Simpson and Joll (1938), Roholm and Teilum (1942) have published cases. The ages of the eight patients were 15, 26, $27,33,34,40,44$, and 44 . The presenting symptom varied in the series: five out of the eight had gynecomastia and complained from the onset of swelling or pain in the breasts; the remainder of pain, discomfort, or a lump in the upper abdomen-i.e., of the causal neoplasm. In three cases there were also reduction in size of the external genitalia-penis, testicles, or both-and loss of libido or potency. Our patient complained of loss of weight and of sweating - and Holl's second patient of obesity. Haemoptysis due to secondary deposits occurred in one case.

Clinical examination revealed gynecomastia in all cases, varying in degree from " as in a girl" to "feminine" size and appearance. Holl's second case had great tenderness. The breasts are described as being nodular or " glandular " to palpation. Holl and Lisser recorded secretions from the nipples. Pigmentation of the areola and hypertrophy of Montgomery follicles are also mentioned. In four of the 